LBL-30113

UCB-PTH-91/2

January 1991

\title{
The Superconformal Master Equation *
}

\author{
A. Giveon ${ }^{\dagger}$, M.B. Halpern, E.B. Kiritsis ${ }^{\ddagger}$, N.A. Obers ${ }^{\S}$ \\ Department of Physics, University of California \\ and \\ Theoretical Physics Group, Lawrence Berkeley Laboratory \\ Berkeley, California 94720
}

\begin{abstract}
We obtain the superconformal master equation, which collects the superconformal solutions of the Virasoro master equation on $g_{x} \times S O(p, q)_{1}$. The associated super C-function and super C-theorem are also obtained. A high-level expansion of the superconformal ansatz $\left(S O(n)_{\operatorname{diag}} \times S O(\operatorname{dim} S O(n))_{1}\right)_{N=1}$ shows a large class of new, generically irrational superconformal field theories with a 3 -form living on the signed graphs of order $n$. The extension to general $\mathrm{N}=2$ superconformal symmetry is also given.
\end{abstract}

Published in Int. J. Mod. Phys. A7 (1992) 947.

*This work was supported in part by the Director, Office of Energy Research, Office of High Energy and Nuclear Physics, Division of High Energy Physics of the U.S. Department of Energy under Contract DE-AC03-76SF00098 and in part by the National Science Foundation under grant PHY85-15857. The work of A.G. is supported in part by a Chaim Weizmann Fellowship.

${ }^{\dagger}$ e-mail: GIVEON@LBL.bitnet, THEORY::GIVEON, GIVEON@CSA3.LBL. Gov

$\ddagger$ e-mail: KIRITSIS@LBL.bitnet, THEORY::KIRITSIS, KIRITSIS@CSA3.LBL.Gov

§e-mail: OBERS@LBL.bitnet, THEORY::OBERS, OBERS@CSA3.LBL.Gov 


\section{Introduction}

In physics, the construction of world sheet fermions [1, 2] played a central role in the discovery of affine Lie algebra $[1,3]$ and superconformal symmetry [2, 4]. Examples of affine-Sugawara constructions [1,5] and coset constructions $[1,5]$ were also given in the first string era, as well as the vertex operator construction of world sheet fermions and $S U(n)_{1}$ from compactified spatial dimensions [6, 7]. The generalization of these constructions $[8,9,10]$ and their application to the heterotic string [11] mark the beginning of the present era. See $[12,13,14]$ for further historical remarks on affine-Virasoro constructions.

The general Virasoro construction on the currents $J_{a}$ of affine $g[15,16,17]$

$$
T(L)=L^{a b}{ }_{*}^{*} J_{a} J_{b} *
$$

systematizes the direct approach used by Bardakci and Halpern $[1,5]$ to obtain the original affine-Sugawara and coset constructions *. The Virasoro master equation [15, $16,17]$ for the inverse inertia tensor $L^{a b}=L^{b a}$ contains the affine-Sugawara nests ${ }^{\dagger}$ and many new conformal constructions $g^{\#}$ on the currents of affine $g$.

In particular, broad classes of exact unitary solutions with irrational central charge [19] have recently been obtained on affine compact $g$. The growing list presently includes the unitary irrational constructions $[19,21,22,23,24]$

$$
\begin{gathered}
\\
\left(\left(\text { simply-laced } g_{x}\right)^{q}\right)_{M}^{\#} \\
S U(3)_{B A S I C}^{\#}=\left\{\begin{array}{c}
S U(3)_{M}^{\#} \\
S U(3)_{D(1)}^{\#}, \quad S U(3)_{D(2)}^{\#}, \quad S U(3)_{D(3)}^{\#} \\
S U(3)_{A(1)}^{\#}, \quad S U(3)_{A(2)}^{\#} \\
S O(2 n)^{\#}[d, 4]^{\#}
\end{array}\right. \\
S O(n)_{\text {diag }}^{\#}=\left\{\begin{array}{c}
S O(5)^{\#}[d, 2] ; \quad S O(2 n+1)^{\#}[d, 6]_{1}, n \geq 3 \\
S O(5)^{\#}[d, 6]_{1} ; \quad S O(2 n+1)^{\#}[d, 6]_{2}, n \geq 3 \\
S O(5)^{\#}[d, 6]_{2}
\end{array}\right.
\end{gathered}
$$

which are obtained for variable level in the BASIC $\supset$ Dynkin $\supset$ Maximal sequence of subansätze, and in $S O(n)_{d i a g}$, the diagonal ansatz on $S O(n)$. The value

$$
c\left(\left(S U(3)_{5}\right)_{D(1)}^{\#}\right)=2\left(1-\frac{1}{\sqrt{61}}\right) \simeq 1.7439
$$

is the lowest unitary irrational central charge yet observed [23]. A very large number [19]

$$
N(g)=2^{n(g)}, \quad n(g)=\operatorname{dim} g(\operatorname{dim} g-1) / 2
$$

of solutions is expected generically on arbitrary level of any $g$, e.g. $N(g) \sim \frac{1}{4}$ billion on $S U(3)$, so the exact constructions in (1.2) are only the first glimpse into a genericallyirrational affine-Virasoro universe of immense new structure.

\footnotetext{
${ }^{*}$ Related ideas are discussed in [18].

$\dagger$ The affine-Sugawara nests [19] include the affine-Sugawara constructions [1, 5, 9], the coset constructions $[1,5,10]$ and the nested coset constructions [20].
} 
Recently, a high-level or semiclassical expansion of the master equation [23] has been developed, which is capable in principle of seeing all high- $k$ smooth unitary constructions on any simple $g$. In an application to the ansatz $S O(n)_{\text {diag }}$, the expansion shows an isomorphism between this subset of unitary affine-Virasoro constructions and the set of graphs of order $n$ [24]. Conversely, the isomorphism provides a Lie group and conformal field theoretic organization of graph theory which may be interesting in mathematics.

The emerging picture, seen most clearly in the isomorphism with graph theory, is that the known rational constructions with Lie group and subgroup symmetry are among those few solutions with the highest symmetry, and the new unitary irrational constructions have at least a residual discrete symmetry, but the generic unitary irrational conformal field theory has no symmetry at all.

In other directions, the master equation has been identified as an Einstein system on the group manifold [16], and a world-sheet action [25] has been obtained for the generic high-level smooth affine-Virasoro construction. Moreover, classical construction of primary fields [25] has been studied in the generic theory. The master equation has also provided an exact C-function and C-theorem [26] on affine-Virasoro space, so that the system may be related to an exact renormalization group equation.

Another important direction is the identification of those new affine-Virasoro constructions which have superconformal symmetry, W-symmetry or other symmetries. In a special case, the question of supersymmetry has been addressed by Mohammedi [27].

In this paper, we obtain the superconformal master equation, which collects the superconformal solutions of the Virasoro master equation on $g_{x} \times S O(p, q)_{1}$. The superconformal system, which possesses an exact super C-function and super C-theorem, is remarkably simple because it is completely general.

We have also found a consistent superconformal ansatz, which we call $\left(S O(n)_{\text {diag }} \times\right.$ $\left.S O(\operatorname{dim} S O(n))_{1}\right)_{N=1}$ because the "bosonic" structure of the ansatz is analogous to that of the ansatz $S O(n)_{\text {diag. }}$. In particular, a high-level expansion of this ansatz shows a large class of new, generically irrational superconformal field theories with a 3-form living on the signed graphs [28] of order $n$. Finally, there are four technical appendices, including the general $\mathrm{N}=2$ construction in Appendix D.

\section{The Virasoro Master Equation}

The general Virasoro construction begins with the currents $J_{a}$ of affine $g[3,1]$,

$$
\left[J_{a}^{(m)}, J_{b}^{(n)}\right]=i f_{a b}^{c} J_{c}^{(m+n)}+m G_{a b} \delta_{m+n, 0}, \quad m, n \in Z
$$

where $f_{a b}^{c}$ and $G_{a b}$ are respectively the structure constants and general Killing metric of $g$. The current algebra (2.1) is completely general since $g$ is not necessarily compact or semi-simple. In particular, to obtain level $x_{I}=2 k_{I} / \psi_{I}^{2}$ of $g_{I}$ in $g=\oplus_{I} g_{I}$ with dual Coxeter number $\tilde{h}_{I}=Q_{I} / \psi_{I}^{2}$, take

$$
G_{a b}=\oplus_{I} k_{I} \eta_{a b}^{I}, f_{a c}{ }^{d} f_{b d}^{c}=-\oplus_{I} Q_{I} \eta_{a b}^{I}
$$


where $\eta_{a b}^{I}$ and $\psi_{I}$ are respectively the Killing metric and highest root of $g_{I}$. The class of operators quadratic in the currents,

$$
T(L) \equiv L_{*}^{a b}{ }_{*}^{*} J_{a} J_{b}^{*} \equiv \sum_{m \in Z} L^{(m)} z^{-m-2}
$$

is defined with symmetric normal ordering, $T_{a b} \equiv{ }_{*}^{*} J_{a} J_{b}{ }_{*}^{*}=T_{b a}$ [15]. The set of coefficients $L^{a b}=L^{b a}$ is called the inverse inertia tensor, in analogy with the spinning top, and the set of all coefficients $\left\{L^{a b}\right\}$ is affine-Virasoro space. The requirement that $T(L)$ is a Virasoro operator

$$
\left[L^{(m)}, L^{(n)}\right]=(m-n) L^{(m+n)}+\frac{c}{12} m\left(m^{2}-1\right) \delta_{m+n, 0}
$$

restricts the values of $L^{a b}$ to those which solve the Virasoro master equation.

The master equation and central charge may be written as $[15,17,26]$

$$
\beta^{a b}(L)=0 \quad, \quad c(L)=2 L^{a b} P_{a b, c d} L^{c d}=2 G_{a b} L^{a b}
$$

where the $\beta$-function $\beta^{a b}$ and metric $P_{a b, c d}$ on affine-Virasoro space are $\ddagger$

$$
\begin{aligned}
\beta^{a b}(L) & \equiv \frac{1}{2} L^{c d} L^{e f} R_{c d, e f}{ }^{a b}-L^{a b} \\
& =-L^{a b}+2 L^{a c} G_{c d} L^{d b}-L^{c d} L^{e f} f_{c e}{ }^{a} f_{d f}^{b}-L^{c d} f_{c e}{ }^{f} f_{d f}{ }^{(a} L^{b) e} \\
P_{a b, c d} & \equiv z^{4}\left\langle T_{a b}(z) T_{c d}(0)\right\rangle=G_{a(c} G_{d) b}-\frac{1}{2} f_{a(c}^{e} f_{d) b}^{f} G_{e f} .
\end{aligned}
$$

The master equation has been identified in [16] as an Einstein-like system on the group manifold.

The corresponding C-function on affine-Virasoro space [26]

$$
\begin{gathered}
A(L)=2 L^{a b} P_{a b, c d}\left(L^{c d}-2 \beta^{c d}(L)\right) \\
\frac{\partial A(L)}{\partial L^{a b}}=-12 P_{a b, c d} \beta^{c d}(L) \\
\left.A(L)\right|_{\beta=0}=c
\end{gathered}
$$

is an action for the Virasoro master equation ${ }^{\S}$. All the conformal field theories of the Virasoro master equation are fixed points of the associated flow

$$
\dot{L}^{a b}=\beta^{a b}(L)
$$

which satisfies the C-theorem [29]

$$
\dot{A}(L) \leq 0
$$

for unitary subflows [26] on positive integer levels of affine compact $g$. It is difficult to resist speculation on the connection between this system and an exact renormalization group equation.

\footnotetext{
${ }^{\ddagger}$ Our notation is $A_{(a} B_{b)} \equiv A_{a} B_{b}+A_{b} B_{a}$ and $A_{[a} B_{b]} \equiv A_{a} B_{b}-A_{b} B_{a}$.

${ }^{\S}$ The C-function equation of motion $P_{a b, c d} \beta^{c d}(L)=0$ in $(2.7 \mathrm{~b})$ is in fact the correct master equation, which follows from the current algebra when there are linear dependences $\xi^{a b} T_{a b}=0$ among the current bilinears $T_{a b}$ on $g_{x}$. This form of the master equation allows continuous solutions $L^{a b} \rightarrow L^{a b}+\xi^{a b}$, and the usual master equation $\beta^{a b}(L)=0$ is a gauge fixing of this symmetry since $\beta^{a b}(L+\xi)=\beta^{a b}-\xi^{a b}$ is easily verified from $P_{a b, c d} \xi^{c d}=0$.
} 


\section{General Superconformal Construction}

The $\mathrm{N}=1$ superconformal algebra is

$$
\begin{gathered}
{\left[L^{(m)}, L^{(n)}\right]=(m-n) L^{(m+n)}+\frac{c}{12} m\left(m^{2}-1\right) \delta_{m+n, 0}} \\
\left\{G^{(r)}, G^{(s)}\right\}=2 L^{(r+s)}+\frac{c}{3}\left(r^{2}-\frac{1}{4}\right) \delta_{r+s, 0} \\
{\left[L^{(m)}, G^{(r)}\right]=\left(\frac{m}{2}-r\right) G^{(m+r)}}
\end{gathered}
$$

where $\left\{G^{(r)}\right\}$ is the supercurrent, with $r \in Z+\frac{1}{2}$ ( Neveu-Schwarz) or $Z$ (Ramond). The equivalent operator product expansion $(\mathrm{OPE})$ is

$$
\begin{gathered}
T(z) T(w)=\frac{c / 2}{(z-w)^{4}}+\left(\frac{2}{(z-w)^{2}}+\frac{1}{z-w} \partial_{w}\right) T(w)+\text { finite } \\
G(z) G(w)=\frac{2 c / 3}{(z-w)^{3}}+\frac{2}{z-w} T(w)+\text { finite } \\
T(z) G(w)=\left(\frac{3 / 2}{(z-w)^{2}}+\frac{1}{z-w} \partial_{w}\right) G(w)+\text { finite }
\end{gathered}
$$

where $T(z)=\sum_{m} L^{(m)} z^{-m-2}$ and $G(z)=\sum_{r} G^{(r)} z^{-r-3 / 2}$. A property of this algebra is that the relations $(3.1 \mathrm{~b}, \mathrm{c})$ or $(3.2 \mathrm{~b}, \mathrm{c})$ imply the Virasoro algebra (3.1a) or (3.2a) via the Jacobi identity

$$
\left[L^{(m)},\left\{G^{(r)}, G^{(s)}\right\}\right]+\left\{G^{(r)},\left[G^{(s)}, L^{(m)}\right]\right\}+\left\{G^{(s)},\left[G^{(r)}, L^{(m)}\right]\right\}=0
$$

so that only the $G G$ and $T G$ algebra is required for superconformal symmetry.

Standard representations of this algebra involve Majorana-Weyl world-sheet fermions

$$
\begin{gathered}
S_{I}(z) S_{J}(w)=\eta_{I J} \Delta(z, w)+{ }_{\circ}^{\circ} S_{I}(z) S_{J}(w) \stackrel{\circ}{\circ}, I, J=1, \cdots, F \\
\Delta(z, w)=\left\{\begin{array}{cc}
\frac{1}{z-w} & (B H-N S) \\
\frac{z+w}{2 \sqrt{z w}(z-w)} & (R)
\end{array}\right.
\end{gathered}
$$

where $S_{I}(z)=\sum_{r} S_{I}^{(r)} z^{-r-1 / 2}, r \in Z+\frac{1}{2}$ (Bardakci-Halpern-Neveu-Schwarz) or $r \in Z$ (Ramond). The fermionic metric $\eta_{I J}$ is the metric on the carrier space of some antisymmetric representation $T$, with $\operatorname{dim} T=F$, of any group $g$, and the fermionic affinization $g_{x(T)}$ has level $x(T)=$ the Dynkin index of $T$. Alternately, and without loss of generality, we may consider the fermions to be in the vector representation of $S O(p, q)_{\tau} \supset g_{x(T)}$, $p+q=F$

$$
J_{I J}=i \sqrt{\frac{\tau \psi^{2}}{2}}{ }_{\circ}^{\circ} S_{I} S_{J} \stackrel{\circ}{\circ}, \quad I<J
$$

with level $\tau=1$ for $F \geq 4$ and $\tau=2$ for $F=3$.

Our construction begins on the manifold

$$
\left(g_{x} \times \text { fermionic } S O(p, q)_{\tau}\right), \quad g_{x} \equiv \oplus_{I} g_{x_{I}}
$$


with a set $J_{A}(A=1, \cdots, \operatorname{dim} g)$ of "bosonic" currents on $g_{x}[3,1]$

$$
\begin{gathered}
J_{A}(z) J_{B}(w)=\frac{G_{A B}}{(z-w)^{2}}+i f_{A B}^{C}\left(\frac{1}{z-w}+\frac{1}{2} \partial_{w}\right) J_{C}(w)+T_{A B}(w)+\mathcal{O}(z-w) \\
J_{A}(z) S_{I}(w)=\text { finite } .
\end{gathered}
$$

Our strategy is to collect those solutions of the Virasoro master equation (2.5) on this manifold which possess at least one supercurrent of the form

$$
G(z)=e^{A I} J_{A}(z) S_{I}(z)+\frac{i}{6} t^{I J K}{ }_{\circ}^{\circ} S_{I}(z) S_{J}(z) S_{K}(z) \stackrel{\circ}{\circ}
$$

whose coefficients $e^{A I}$ and $t^{I J K}$ will be called the vielbein and 3 -form respectively. The Neveu-Schwarz copy of the supercurrent in (3.8) is the most general spin 3/2 quasiprimary field which can be constructed from the fermions and the currents; addition of $\partial S_{I}$ terms to the supercurrent is discussed in Appendix C.

The GG OPE (3.2b) defines the stress tensor in the fermionic basis

$$
\begin{aligned}
T(z) & =\mathcal{L}^{A B} T_{A B}+\mathcal{F}^{I J}\left({ }_{\circ}^{\circ} S_{I}(z) \stackrel{\leftrightarrow}{\partial}_{z} S_{J}(z) \stackrel{\circ}{\circ}-\frac{\epsilon \eta_{I J}}{4 z^{2}}\right) \\
& +i \mathcal{M}^{I J A} J_{A}(z){ }_{\circ}^{\circ} S_{I}(z) S_{J}(z) \stackrel{\circ}{\circ}+\mathcal{R}^{I J K L}{ }_{\circ}^{\circ} S_{I}(z) S_{J}(z) S_{K}(z) S_{L}(z) \stackrel{\circ}{\circ}
\end{aligned}
$$

where

$$
A \overleftrightarrow{\partial} B \equiv A(\partial B)-(\partial B) A, \quad \epsilon \equiv\left\{\begin{array}{cc}
0 & (B H-N S) \\
1 & (R)
\end{array}\right.
$$

and the coefficients of the stress tensor are determined as

$$
\begin{gathered}
\mathcal{L}^{A B}=\mathcal{L}^{A B}(e, t) \equiv \frac{1}{2} e^{A I} e^{B J} \eta_{I J} \\
\mathcal{F}^{I J}=\mathcal{F}^{I J}(e, t) \equiv-\frac{1}{4} e^{A I} e^{B J} G_{A B}-\frac{1}{8} t^{I K L} t^{J M N} \eta_{K M} \eta_{L N} \\
\mathcal{M}^{I J A}=\mathcal{M}^{I J A}(e, t) \equiv \frac{1}{2} e^{B I} e^{C J} f_{B C}{ }^{A}+\frac{1}{2} t^{I J K} e^{A L} \eta_{K L} \\
\mathcal{R}^{I J K L}=\mathcal{R}^{I J K L}(e, t) \equiv-\frac{1}{24} t^{M I[J} t^{K L] N} \eta_{M N}
\end{gathered}
$$

Moreover, the forms

$$
c=2 \mathcal{L}^{A B} G_{A B}-2 \mathcal{F}^{I J} \eta_{I J}=\frac{3}{2} e^{A I} e^{B J} G_{A B} \eta_{I J}+\frac{1}{4} t^{I J K} t^{L M N} \eta_{I L} \eta_{J M} \eta_{K N}
$$

are obtained for the superconformal central charge.

The TG OPE, computed with (3.8) and (3.9), is more involved. The complete set of relations is

$$
\begin{aligned}
\frac{3}{2} e^{A I}= & \mathcal{L}^{B C} e^{D I} M_{B C, D}{ }^{A}+\left(-2 \mathcal{F}^{I J} e^{A K}-2 \mathcal{M}^{I J B} e^{C K} f_{B C}{ }^{A}+\mathcal{M}^{M J A} t^{N K I} \eta_{M N}\right) \eta_{J K} \\
& \frac{3}{2} t^{I J K}=2 e^{A[K} \mathcal{M}^{I J] B} G_{A B}-2\left(\mathcal{F}^{L[K} t^{I J] M}+6 \mathcal{R}^{N L[I J} t^{K] R M} \eta_{N R}\right) \eta_{L M}
\end{aligned}
$$


where $A^{[I J} B^{K]} \equiv A^{I J} B^{K}+A^{J K} B^{I}+A^{K I} B^{J}$, which is totally antisymmetric in $I J K$ when $A^{I J}$ is antisymmetric, and

$$
\begin{gathered}
M_{A B, C}{ }^{D} \equiv P_{A B, C E} G^{E D} \\
P_{A B, C D}=z^{4}<T_{A B}(z) T_{C D}(0)>=G_{A(C} G_{D) B}-\frac{1}{2} f_{A(C}{ }^{E} f_{D) B}{ }^{F} G_{E F} .
\end{gathered}
$$

Possible constraints, such as those necessary to eliminate $S_{I}$ terms in $T G$, are automatically implied when both (3.11) and (3.13) are satisfied.

According to our previous argument, the $G G$ relations (3.11) and the $T G$ relations (3.13) are a complete set for (at least) $\mathrm{N}=1$ superconformal symmetry, guaranteeing in particular that the Virasoro master equation

$$
\beta^{a b}(L(e, t))=0, \quad G_{a b}=\left(\begin{array}{cc}
G_{A B} & 0 \\
0 & G_{I J, K L}
\end{array}\right), \quad f_{a b}^{c}=\left(\begin{array}{cc}
f_{A B}^{C} & 0 \\
0 & f_{I J, K L} M N
\end{array}\right)
$$

is satisfied on $g_{x} \times S O(p, q)_{\tau}$, where $L^{a b}(e, t)$ are the stress tensor coefficients (3.11), expressed in the current-current basis (see Appendix A).

A compact summary of the construction is the superconformal master equation (SME)

$$
\begin{aligned}
2 E^{A I}(e, t) \equiv & -e^{A I}+e^{B J} e^{C K} e^{D I}\left(\delta_{B}^{A} G_{C D}+f_{E B}{ }^{A} f_{C D}{ }^{E}\right) \eta_{J K} \\
& +t^{I J K}\left(\frac{1}{2} t^{M N L} e^{A R} \eta_{L R}+e^{B M} e^{C N} f_{B C}{ }^{A}\right) \eta_{J M} \eta_{K N}=0 \\
2 T^{I J K}(e, t) \equiv & -t^{I J K}+e^{A L} e^{B[K} t^{I J] M} G_{A B} \eta_{L M}+2 e^{A I} e^{B J} e^{C K} f_{A B}^{D} G_{D C} \\
& +\left(\frac{1}{2} t^{P[I J} t^{K] M N} t^{R L Q}+2 t^{M P I} t^{N Q J} t^{L R K}\right) \eta_{P Q} \eta_{M R} \eta_{N L}=0 \\
c= & \frac{3}{2} e^{A I} e^{B J} G_{A B} \eta_{I J}+\frac{1}{4} t^{I J K} t^{L M N} \eta_{I L} \eta_{J M} \eta_{K N}
\end{aligned}
$$

which is obtained from (3.13) by eliminating the stress tensor coefficients (3.11) in favor of the vielbein $e^{A I}$ and the 3 -form $t^{I J K}$ of the supercurrent $~$. Note that each solution $(e, t)$ of the SME automatically gives a Neveu-Schwarz and a Ramond copy of the superconformal system, as expected.

Remarkably, the SME (3.16) is a "consistent ansatz" [19] of the Virasoro master equation, in that the superconformal system contains the same number of (cubic) equations as unknowns, so that we may count the generically-expected number of superconformal constructions (see Section 5). Unitary solutions of the SME are recognized when the vielbein and 3 -form are real in any Cartesian basis of compact $g_{x} \times S O(F)_{\tau}, x_{I} \in N$. We also remark that the K-conjugate construction $[1,5,10,15] \tilde{L}=L_{g}-L$ of a superconformal construction $L$, although a commuting Virasoro operator, is not generally superconformal.

The SME contains the known superconformal constructions listed in Appendix B, extension to include linear fermionic terms in the supercurrent is discussed in Appendix $\mathrm{C}$, and the $\mathrm{N}=2$ superconformal construction is summarized in Appendix D.

IThe special case treated by Mohammedi [27] may be obtained from the SME by imposing the conditions $\mathcal{M}^{I J A}(e, t)=\mathcal{R}^{I J K L}(e, t)=0$ as additional constraints. 


\section{The Super C-Function}

We have found a first-order action for the SME

$$
\begin{aligned}
A_{1}(e, t, \mathcal{L}, \mathcal{F}, \mathcal{M}, \mathcal{R})=3 e^{A I} e^{B J} G_{A B} \eta_{I J}+\frac{1}{2} t^{I J K} t^{L M N} \eta_{I L} \eta_{J M} \eta_{K N} \\
\quad+2 \mathcal{L}^{A B}\left(\mathcal{L}^{C D}-2 \mathcal{L}^{C D}(e, t)\right) P_{A B, C D}+8 \mathcal{F}^{I J}\left(\mathcal{F}^{K L}-2 \mathcal{F}^{K L}(e, t)\right) \eta_{I K} \eta_{J L} \\
\quad+4 \mathcal{M}^{I J A}\left(\mathcal{M}^{K L B}-2 \mathcal{M}^{K L B}(e, t)\right) G_{A B} \eta_{I K} \eta_{J L} \\
\quad+48 \mathcal{R}^{I J K L}\left(\mathcal{R}^{M N P Q}-2 \mathcal{R}^{M N P Q}(e, t)\right) \eta_{I M} \eta_{J N} \eta_{K P} \eta_{L Q}
\end{aligned}
$$

where $\mathcal{L}(e, t), \mathcal{F}(e, t), \mathcal{M}(e, t)$ and $\mathcal{R}(e, t)$ are the stress tensor coefficients defined in (3.11) and $\mathcal{L}, \mathcal{F}, \mathcal{M}, \mathcal{R}$ are Lagrange multipliers. The $A_{1}$ equations of motion for the Lagrange multipliers are the stress tensor defining relations (3.11), which followed from the $G G \mathrm{OPE}$, and the equations of motion for $e^{A I}$ and $t^{I J K}$ are the $T G$ relations in (3.13).

Substitution of the $G G$ defining relations (3.11) into the first-order action gives the action $A(e, t)$ for the fundamental variables $e^{A I}$ and $t^{I J K}$

$$
\begin{aligned}
A(e, t) & =\frac{3}{2} e^{A I}\left(e^{B J}-2 E^{B J}(e, t)\right) G_{A B} \eta_{I J} \\
& +\frac{1}{4} t^{I J K}\left(t^{L M N}-2 T^{L M N}(e, t)\right) \eta_{I L} \eta_{J M} \eta_{K N} .
\end{aligned}
$$

The functions $E^{A I}(e, t)$ and $T^{I J K}(e, t)$ are defined in (3.16), and it is easily verified that

$$
\begin{gathered}
E^{A I}(e, t)=-\frac{1}{12} G^{A B} \eta^{I J} \frac{\partial A(e, t)}{\partial e^{B J}} \\
T^{I J K}(e, t)=-\frac{1}{2} \eta^{I L} \eta^{J M} \eta^{K N} \frac{\partial A(e, t)}{\partial t^{L M N}}
\end{gathered}
$$

so the SME $E^{A I}=T^{I J K}=0$ follows directly from this action.

Comparison with the central charge in $(3.16 \mathrm{c})$ shows that

$$
\left.A(e, t)\right|_{E=T=0}=c
$$

so the action $A(e, t)$ is a super $\mathrm{C}$-function on $\mathrm{N}=1$ affine-Virasoro space.

All the superconformal field theories of the SME are fixed points of the associated flow

$$
\dot{e}^{A I}=E^{A I}(e, t) \quad, \quad \dot{t}^{I J K}=T^{I J K}(e, t)
$$

whose vector fields $E^{A I}(e, t)$ and $T^{I J K}(e, t)$ in (3.16) will be called the $\beta$-functions of the supercurrent. Unitary subflows are obtained when the Cartesian initial values $e^{A I}(0)$ and $t^{I J K}(0)$ are real on positive integer levels of compact $g_{x} \times S O(F)_{\tau}$, and the super C-theorem

$$
\dot{A}(e, t)=-\left(12 E^{A I} E^{B J} G_{A B} \eta_{I J}+2 T^{I J K} T^{L M N} \eta_{I L} \eta_{J M} \eta_{K N}\right) \leq 0
$$

follows for unitary subflows. 
The associated stress tensor flow $\left\{\dot{\mathcal{L}}^{A B}(e, t), \quad \dot{\mathcal{M}}^{I J A}(e, t), \quad \dot{\mathcal{F}}^{I J}(e, t)\right.$, $\left.\dot{\mathcal{R}}^{I J K L}(e, t)\right\}$, defined by chain rule, is the closed subflow

$$
\dot{L}^{a b}(e, t)=\beta^{a b}(L(e, t))
$$

of the stress tensor flow (2.8) in the current-current basis of $g_{x} \times S O(p, q)_{\tau}$. The implied relation between the stress tensor $\beta$-function $\beta^{a b}(L)$ in (2.6a) and the $\beta$-functions (4.5) of the supercurrent

$$
\beta^{a b}(L(e, t))=\frac{\partial L^{a b}(e, t)}{\partial e^{A I}} E^{A I}(e, t)+\frac{\partial L^{a b}(e, t)}{\partial t^{I J K}} T^{I J K}(e, t)
$$

can be verified from the explicit form of $\left\{L^{a b}(e, t)\right\}$ in Appendix A. Off criticality, the G,T flow system (4.5) and (4.7) maintains the GG OPE (3.2b), so that global supersymmetry $\left\{G^{(0)}, G^{(0)}\right\}=2\left[L^{(0)}-\frac{c}{24}\right]$ is maintained $\|$ throughout the flow, where the constant $c$ is defined in (3.12).

We finally remark that the super C-function can be simply expressed in terms of the stress tensor coefficients (3.11) as

$$
\begin{gathered}
A(e, t)=\mathcal{L}^{A B}\left(4 G_{A B}-2 P_{A B, C D} \mathcal{L}^{C D}\right)-\mathcal{F}^{I J}\left(4 \eta_{I J}+8 \mathcal{F}_{I J}\right) \\
-4 \mathcal{M}^{I J A} \mathcal{M}_{I J A}-48 \mathcal{R}^{I J K L} \mathcal{R}_{I J K L}
\end{gathered}
$$

where indices are lowered with $G_{A B}$ and $\eta_{I J}$. The form in the current-current basis $\left\{L^{a b}(e, t)\right\}$ on $g_{x} \times S O(p, q)_{\tau}$

$$
A(e, t)=4 G_{a b} L^{a b}-2 L^{a b} P_{a b, c d} L^{c d}=2 G_{a b}\left(L^{a b}-\beta^{a b}(L)\right)
$$

is even simpler, and should be compared with the general C-function in (2.7a).

"The first-order action $A_{1}$, which is also a super C-function, defines another $\mathrm{G}, \mathrm{T}$ flow which violates global supersymmetry. 


\section{$5 \quad$ Automorphisms and Counting}

The automorphism group $A u t(S, J)$ of the fermionic algebra (3.4) and the current algebra $(3.7 \mathrm{a})$ is

$$
\begin{aligned}
\left(S_{I}\right)^{\prime}= & \Omega_{I}{ }^{J} S_{J},\left(J_{A}\right)^{\prime}=\omega_{A}^{B} J_{B} \\
\omega_{A}{ }^{C} G_{C D} \omega_{B}{ }^{D}= & G_{A B}, \omega_{A}{ }^{D} \omega_{B}{ }^{E} \omega_{C}{ }^{F} f_{D E F}=f_{A B C} \\
& \Omega_{I}{ }^{K} \eta_{K L} \Omega_{J}{ }^{L}=\eta_{I J}
\end{aligned}
$$

so that $\omega \in \operatorname{Aut}(g), \Omega \in O(p, q)$ and $\operatorname{Aut}(S, J) \subset \operatorname{Aut}(g \times S O(p, q))$. Correspondingly, the super C-function (4.2) is invariant under the automorphisms

$$
\begin{gathered}
\left(e^{\prime}\right)^{A I}=e^{B J}\left(\omega^{-1}\right)_{B}{ }^{A}\left(\Omega^{-1}\right)_{J}{ }^{I} \\
\left(t^{\prime}\right)^{I J K}=t^{L M N}\left(\Omega^{-1}\right)_{L}{ }^{I}\left(\Omega^{-1}\right)_{M}{ }^{J}\left(\Omega^{-1}\right)_{N}{ }^{K} .
\end{gathered}
$$

It follows from (4.3) that the SME (3.16) transforms covariantly under (5.2), so that $\left(e^{\prime}, t^{\prime}\right)$ and $(e, t)$ are automorphically-equivalent superconformal field theories, with the same spectrum and central charge.

The SME (3.16) is a set of $F(\operatorname{dim} g+(F-1)(F-2) / 6)$ coupled cubic equations on an equal number of unknowns $e^{A I}$ and $t^{I J K}$. Gauge-fixing the inner automorphisms of $g \times S O(p, q)$ will reduce the system by $\operatorname{dim} g+F(F-1) / 2$ equations and unknowns, so that

$$
N_{s}=3^{n_{s}}, n_{s}=(F-1) \operatorname{dim} g+\frac{1}{6} F(F-1)(F-5)
$$

superconformal field theories are expected generically on each level of $g_{x} \times S O(p, q)_{\tau}$. For comparison, the total number of (not necessarily superconformal) affine-Virasoro constructions [19]

$$
N=2^{n}, \quad n=\frac{\operatorname{dim} g(\operatorname{dim} g-1)}{2}+\frac{1}{8} F\left(F^{2}-1\right)(F-2)
$$

is expected generically on each level of the same manifold, after gauge-fixing the inner automorphisms. The numbers of distinct Virasoro and superconformal constructions can be less due to residual automorphisms [21, 23, 24] and/or non-generic behavior, such as quadratic deformations. It is clear from (5.3) and (5.4) that superconformal constructions, although copious, are relatively rare on large groups since the superconformal fraction $N_{s} / N$ is exponentially small in this case. 


\section{The Ansatz $\left(\mathrm{SO}(\mathbf{n})_{\operatorname{diag}} \times \mathrm{SO}(\operatorname{dim} \mathrm{SO}(\mathbf{n}))_{\tau}\right)_{\mathrm{N}=1}$}

Consistent ansätze $[19,21,23,24]$ have played a central role in solving the Virasoro master equation, and many consistent superconformal ansätze are possible for the SME. For example, the consistent fermionic superconformal ansatz

$$
e^{A I}=0, \quad G=\frac{i}{6} t^{I J K} \circ S_{I} S_{J} S_{K} \stackrel{\circ}{\circ}
$$

contains the known non-linear supersymmetry [30] and a large number of new purely fermionic superconformal constructions. We have also found a consistent superconformal ansatz whose "bosonic" structure is analogous to the ansatz $S O(n)_{\text {diag }}[24]$ in which the isomorphism with graph theory was observed.

The ansatz, which we call $\left(S O(n)_{d i a g} \times S O(\operatorname{dim} S O(n))_{\tau}\right)_{N=1}$, is

$$
\begin{gathered}
e^{i j, k l}=\Lambda_{i j} \delta_{i k} \delta_{j l} \\
t^{i j, i l, j l}=t(i j l) f^{i j, i l, j l} \\
G=\sum_{i<j} \Lambda_{i j} J_{i j} S_{i j}-i \sqrt{\frac{\tau \psi^{2}}{2}} \sum_{i<j<l} t(i j l) \circ_{\circ}^{\circ} S_{i j} S_{i l} S_{j l} \stackrel{\circ}{\circ}
\end{gathered}
$$

where we have taken $A=I=(i, j), 1 \leq i<j \leq n$ for the "bosonic" $S O(n)$ and the fermionic $S O(\operatorname{dim} S O(n)), \psi$ is the highest root of $S O(n)$, and $f^{i j, k l, m n}$ are the structure constants of $S O(n)$ in the physicist's standard basis with $\eta_{i j, k l}=\delta_{i k} \delta_{j l}$ (see Appendix A). Note that the vielbein $e^{A I}$ is diagonal, and that we have taken the 3 -form $t^{I J K}$ non-zero only when the structure constants $f^{I J K}$ are non-zero. The reduced 3 -form $t(i j l)$ is valued on unordered index triplets $(i j l)$, so all other non-zero $t^{i j, k l, r s}$ may be obtained from $(6.2 \mathrm{~b})$ by antisymmetry of the structure constants. Consistency of the ansatz depends on two properties of the structure constants in this basis

$$
\begin{gathered}
f_{i j, k l}{ }^{m n} f_{i j, k l}{ }^{r s \neq m n}=0, \forall i j, k l \\
f_{i j, k l}^{m n} f_{k l, r s}{ }^{t u} f_{r s, i j}{ }^{v w}=0, \forall i j, k l, r s \text { when } f^{m n, t u, v w} \neq 0
\end{gathered}
$$

the first of which was necessary for the consistency of the ansatz $S O(n)_{\text {diag }}$.

The reduced SME

$$
\begin{gathered}
\Lambda_{i j}=k \Lambda_{i j}^{3}+\frac{\tau \psi^{2}}{2} \sum_{l \neq i, j}\left[\Lambda_{i j}\left(\Lambda_{i l}^{2}+\Lambda_{j l}^{2}+t^{2}(i j l)\right)+2 \Lambda_{i l} \Lambda_{j l} t(i j l)\right] \\
t(i j l)=k\left(\Lambda_{i j}^{2}+\Lambda_{i l}^{2}+\Lambda_{j l}^{2}\right) t(i j l)+2 k \Lambda_{i j} \Lambda_{i l} \Lambda_{j l}+\frac{\tau \psi^{2}}{2} t^{3}(i j l) \\
+\frac{\tau \psi^{2}}{2} \sum_{m \neq i, j, l}\left[t(i j l)\left(t^{2}(i j m)+t^{2}(i l m)+t^{2}(j l m)\right)-2 t(i j m) t(i l m) t(j l m)\right] \\
c=\frac{3 \psi^{2}}{4}\left[x \sum_{i<j} \Lambda_{i j}^{2}+\tau \sum_{i<j<l} t^{2}(i j l)\right], \quad x=\frac{2 k}{\psi^{2}}
\end{gathered}
$$


is obtained for the ansatz, where the symmetry convention

$$
\Lambda_{i j}=\Lambda_{j i}, \quad i \neq j \quad\left(\Lambda_{i i}=0\right)
$$

has been adopted to simplify the summations. A complete set of equations is obtained by choosing $i<j$ in (6.4a) and $i<j<l$ in $(6.4 \mathrm{~b})$. The corresponding non-zero coefficients of the stress tensor are

$$
\begin{gathered}
\mathcal{L}^{i j, k l}=\frac{1}{2} \Lambda_{i j}^{2} \delta_{i k} \delta_{j l} \\
\mathcal{F}^{i j, k l}=-\frac{1}{4}\left(k \Lambda_{i j}^{2}+\frac{\tau \psi^{2}}{2} \sum_{l} t^{2}(i j l)\right) \delta_{i k} \delta_{j l} \\
\mathcal{M}^{i l, j l, i j}=\frac{1}{2}\left(\Lambda_{i l} \Lambda_{j l}+\Lambda_{i j} t(i j l)\right) f^{i j, i l, j l} \\
\mathcal{R}^{i j, k l, m n, r s}=-\frac{1}{24} \sum_{p<q} t^{p q, i j[k l} t^{m n, r s] p q}
\end{gathered}
$$

which shows in particular that the "bosonic" coefficient $\mathcal{L}^{A B}$ in (6.6a) has the same diagonal form as in the ansatz $S O(n)_{\text {diag }}$.

The consistent ansatz (6.4) contains

$$
N_{s}=3^{n\left(n^{2}-1\right) / 6}
$$

superconformal constructions generically, which includes copies of those constructions on $S O(n)_{x} \times S O(\operatorname{dim} S O(n))_{\tau}$ in Appendix B whose "bosonic" subgroups are restricted to

$$
h\left(S O(n)_{\text {diag }}\right) \equiv \times_{i=1}^{M} S O\left(m_{i}\right), \quad \sum_{i=1}^{M} m_{i}=n
$$

or the KS analogue $h\left(S O(n)_{\text {diag }}\right)^{K S}$. Each factor $S O\left(m_{i} \geq 4\right)$ is regularly embedded in $S O(n)_{x}$, while $S O(3)$ factors are embedded at level $2 x$. The number of solutions in the ansatz may be less than (6.7) due to non-generic behavior, and the number of physically distinct constructions is certainly less than (6.7) due to the residual automorphisms of the ansatz

$$
\begin{gathered}
\Lambda_{i j}^{\prime}=\sigma_{\pi(i) \pi(j)} \Lambda_{\pi(i) \pi(j)}=\left(\omega\left(\Lambda \Omega^{-1}\right) \omega^{-1}\right)_{i j} \\
t^{\prime}(i j l)=\sigma_{\pi(i) \pi(j)} \sigma_{\pi(i) \pi(l)} \sigma_{\pi(j) \pi(l)} t(\pi(i) \pi(j) \pi(l))=\left(t(\omega \Omega)^{-1}(\omega \Omega)^{-1}(\omega \Omega)^{-1}\right)(i j l)
\end{gathered}
$$

where $\sigma_{i j}=\sigma_{j i} \in\{1,-1\}$ and $\pi(i)$ is any permutation of the $S O(n)$ vector indices $i$. The automorphisms in (6.9) are composed as follows. First apply a fermionic automorphism

$$
\Omega_{i j, k l}(\sigma)=\sigma_{i j} \delta_{i k} \delta_{j l} \in O(\operatorname{dim} S O(n))
$$

which induces possible sign changes in any subset of $\Lambda_{i j}$ 's, and then apply an indexpermutation

$$
\omega(\pi) \in S O(n)_{V}
$$

which lives in the vector subgroup $S O(n)_{V}$ of $S O(n) \times O(\operatorname{dim} S O(n))$.

The simplest case is $\left(S O(3)_{x} \times S O(3)_{2}\right)_{N=1}$,

$$
\Lambda_{12}=\Lambda_{12}\left[k \Lambda_{12}^{2}+\psi^{2}\left(\Lambda_{13}^{2}+\Lambda_{23}^{2}+t^{2}\right)\right]+2 \psi^{2} t \Lambda_{13} \Lambda_{23}
$$




$$
\begin{gathered}
\Lambda_{13}=\Lambda_{13}\left[k \Lambda_{13}^{2}+\psi^{2}\left(\Lambda_{23}^{2}+\Lambda_{12}^{2}+t^{2}\right)\right]+2 \psi^{2} t \Lambda_{23} \Lambda_{12} \\
\Lambda_{23}=\Lambda_{23}\left[k \Lambda_{23}^{2}+\psi^{2}\left(\Lambda_{12}^{2}+\Lambda_{13}^{2}+t^{2}\right)\right]+2 \psi^{2} t \Lambda_{12} \Lambda_{13} \\
t=k\left(\Lambda_{12}^{2}+\Lambda_{13}^{2}+\Lambda_{23}^{2}\right) t+2 k \Lambda_{12} \Lambda_{13} \Lambda_{23}+\psi^{2} t^{3} \\
c=\frac{3}{2}\left[k\left(\Lambda_{12}^{2}+\Lambda_{13}^{2}+\Lambda_{23}^{2}\right)+\psi^{2} t^{2}\right]
\end{gathered}
$$

where $t \equiv t(123)$, which contains 81 solutions generically. Although we have not solved this case exhaustively, we have verified that the following known constructions are included: one copy of $\mathrm{G}=\mathrm{T}=0$, eight automorphically equivalent copies of the $\mathrm{N}=1$ affineSugawara construction on $g=S O(3)$, twelve equivalent copies of the KS coset construction $S O(3) / S O(2)$, two copies of the non-linear SUSY on $S O(3)$, and eight copies of the GKO N=1 coset construction $\left(S O(3)_{x} \times S O(3)_{2}\right) / S O(3)_{x+2}$.

We also find twelve copies of a superconformal quadratic deformation, which we call $\operatorname{Cartan}\left(S O(3)_{x} \times S O(3)_{2}\right)_{N=1}^{\#}$

$$
\begin{gathered}
\Lambda_{13}=\Lambda_{23}=0, \quad \Lambda_{12}=\frac{1}{\sqrt{k}} \sqrt{1-\psi^{2} \tilde{t}^{2} / k}, \quad t=\frac{1}{\sqrt{k}} \tilde{t} \\
0 \leq \tilde{t} \leq \sqrt{\frac{x}{2}}, \quad c=\frac{3}{2}
\end{gathered}
$$

whose parameter range is fixed as shown by unitarity. As special points, this deformation contains the $\mathrm{N}=1$ affine-Sugawara construction in (B.1) on $S O(2) \times(S O(1) \equiv$ one free fermion) and the KS subgroup construction in (B.4) with $h^{K S}=S O(2)$. The stress tensor of this deformation is $\operatorname{Cartan}\left(S O(3)_{x} \times S O(3)_{2}\right)^{\#}[19]($ at $\mathrm{c}=1)$ plus a free fermion term, so the construction is presumably related to the line of $Z_{2}$ orbifolds $\times$ one free fermion.

\section{High-level Expansions}

High-level expansion [23] has been used to see and classify large numbers of solutions of the Virasoro master equation on simple $g$, viz. the isomorphism with graph theory in the ansatz $S O(n)_{\text {diag }}$ [24]. On semi-simple $g$, a variety of such expansions is possible due to the presence of various levels $x_{I}$, and only the simplest case, $x_{I} \rightarrow \infty, \forall I$ with $x_{I} / x_{J}$ fixed [21], has been noted so far.

These expansions have been relatively simple because the theories are effectively abelianized in these limits. Expansions with some levels fixed, e.g. the fixed fermionic level $\tau=1,2$ in the SME, will be generally more complex because some of the high-level dynamics remains non-abelian.

At least two high-level expansions

$$
\begin{gathered}
e^{A I}=\frac{1}{\sqrt{k}} \sum_{p=0}^{\infty} e_{(p)}^{A I} k^{-p}, \quad t^{I J K}=\frac{1}{\sqrt{k}} \sum_{p=0}^{\infty} t_{(p)}^{I J K} k^{-p} \\
e^{A I}=\frac{1}{k} \sum_{p=0}^{\infty} e_{(p)}^{A I} k^{-p}, \quad t^{I J K}=\sum_{p=0}^{\infty} t_{(p)}^{I J K} k^{-p}
\end{gathered}
$$


are possible for the SME on simple $g_{x}$ with $G_{A B}=k \eta_{A B}, x=2 k / \psi^{2}$, both of which correspond to integer powers of $k^{-1}$ for the stress tensor. The class of solutions (7.1a) is vielbein-dominated in the sense that the leading equation

$$
e_{(0)}^{A I}=k e_{(0)}^{A J} e_{(0)}^{C K} e_{(0)}^{D I} \eta_{C D} \eta_{J K}
$$

involves only the vielbein, while the equation for the leading term $t_{(0)}^{I J K}$ of the 3 -form depends on $e_{(0)}^{A I}$. According to Appendix B, this class contains the $\mathrm{N}=1$ affine-Sugawara and KS constructions, and the deformation (6.13) was parametrized to fall in this class. In contrast, the $\mathrm{GKO} N=1$ constructions are found in class (7.1b), while the fermionic constructions $e^{A I}=0$ are not accessible to high-level analysis, although large $F$ methods may be helpful. 


\section{Superconformal Constructions on Signed Graphs}

As an example, we study the vielbein-dominated high-level expansion (7.1a)

$$
\begin{gathered}
\Lambda_{i j}=\frac{1}{\sqrt{k}} \sum_{p=0}^{\infty} \Lambda_{i j}^{(p)} k^{-p}, t(i j l)=\frac{1}{\sqrt{k}} \sum_{p=0}^{\infty} t^{(p)}(i j l) k^{-p}, c=\sum_{p=0}^{\infty} c_{p} k^{-p} \\
G=\frac{1}{\sqrt{k}}\left(\sum_{i<j} \Lambda_{i j}^{(0)} J_{i j} S_{i j}-i \sqrt{\frac{\tau \psi^{2}}{2}} \sum_{i<j<l} t^{(0)}(i j l){ }_{\circ}^{\circ} S_{i j} S_{i l} S_{j l} \stackrel{\circ}{\circ}\right)+\mathcal{O}\left(k^{-3 / 2}\right)
\end{gathered}
$$

of the ansatz $\left(S O(n)_{\text {diag }} \times S O(\operatorname{dim} S O(n))_{\tau}\right)_{N=1}$ in $(6.4)$.

We focus first on the zeroth order equation $\Lambda_{i j}^{(0)}=\left(\Lambda_{i j}^{(0)}\right)^{3}$ for the vielbein, whose solution

$$
\begin{gathered}
\Lambda_{i j}^{(0)}=\Theta_{i j} \quad, \quad \Theta_{i j} \in\{0,1,-1\} \\
c_{0}=\frac{3}{2} \sum_{i<j} \Theta_{i j}^{2}
\end{gathered}
$$

shows the basic unit $3 / 2$ for $N=1$ high-level central charges. The structure $\Theta_{i j}$ is the adjacency matrix for any signed [28] labelled graph of order $n$, according to the graphrules

$$
\begin{aligned}
& S O(n) \text { vector-indices } i \leftrightarrow \text { points } i \text { in directed graph } G \\
& \qquad \Theta_{i j}= \pm 1 \leftrightarrow \text { line with a } \pm \text { between } i \text { and } j .
\end{aligned}
$$

These are the $3^{\left(\begin{array}{c}n \\ 2\end{array}\right)}$ signed labelled graphs of $\left(S O(n)_{\text {diag }} \times S O(\operatorname{dim} S O(n))_{\tau}\right)_{N=1}$, whose structure, except for the signs, is that of the labelled graphs of $S O(n)_{\text {diag }}$ [24]. As an illustration, the signed labelled complete graphs of $\left(S O(3)_{\text {diag }} \times S O(3)_{2}\right)_{N=1}$ are shown in Fig. 1.

We also remark that

$$
\Theta_{i j}^{2}=\theta_{i j} \in\{0,1\}
$$

where $\theta_{i j}$ is the adjacency matrix of the corresponding labelled (unsigned) graph of order $n$, and that, according to (6.6a) and (8.4), the leading term of the "bosonic" stress tensor coefficient $\mathcal{L}^{A B}$

$$
\mathcal{L}^{i j, k l}=\frac{\theta_{i j}}{2 k} \delta_{i k} \delta_{j l}+\mathcal{O}\left(k^{-2}\right)
$$

has the form of $S O(n)_{\operatorname{diag}}[24]$.

As in $S O(n)_{\text {diag }}$, the graph theory naturally reflects the residual automorphisms (6.9) of the ansatz. According to the fermionic automorphism (6.10), the sign choice $\Theta= \pm 1$ for any particular graph edge is automorphically equivalent, so we may choose $\Theta=+1$ for all edges. This is the gauge condition

$$
\Theta_{i j}=\theta_{i j}
$$

where $\theta_{i j}$ is the adjacency matrix of the corresponding labelled (unsigned) graph. Then, the permutation automorphism (6.11)

$$
\theta_{i j}^{\prime}=\theta_{\pi(i) \pi(j)}=\left(\omega \theta \omega^{-1}\right)_{i j}, \quad \omega \in S O(n)_{V}
$$


shows that graph relabellings are automorphically equivalent [24] as well. The complete gauge fixing is

$$
\text { signed labelled graphs } \underset{\text { gauge fix }}{\longrightarrow} \text { graphs (unsigned, unlabelled) }
$$

so the physically distinct solutions of the ansatz $\left(S O(n)_{\text {diag }} \times S O(\operatorname{dim} S O(n))_{\tau}\right)_{N=1}$ are constructed on the set of graphs of order $n$. As shown in the example of Fig. 1, the signed labelled complete graphs of $\left(S O(3)_{\text {diag }} \times S O(3)_{2}\right)_{N=1}$ are gauge fixed to the complete graph of order 3 .

In order to identify their graphs ${ }^{* *}$, we have computed the high-level form of the relevant known constructions in Appendix B, and the deformation in (6.13):

1. The $\mathrm{N}=1$ affine-Sugawara construction (B.1) on $S O(n)_{x} \times S O(\operatorname{dim} S O(n))_{\tau}$ lives on the complete graph $K_{n}$ with

$$
t^{(0)}(i j l)=-1, \quad \forall i, j, l .
$$

2. The $\mathrm{N}=1 \mathrm{KS}$ coset constructions (B.5) on

$$
\frac{S O(n)_{x} \times S O(\operatorname{dim} S O(n))_{\tau}}{h\left(S O(n)_{\text {diag }}\right)^{K S} \times S O\left(\operatorname{dim} h\left(S O(n)_{\text {diag }}\right)\right)}
$$

live on the fundamental coset graphs [24], or complete $M$-partite graphs of order $n$, shown in Fig. 2, with

$$
t^{(0)}(i j l)=\left\{\begin{array}{cc}
-1 & \text { when } i, j, l \\
0 & \text { are in distinct lacunae }
\end{array} .\right.
$$

3. The $\mathrm{N}=1$ affine-Sugawara constructions (B.1) on the subgroups $S O(n)_{\operatorname{diag}} \times S O\left(\operatorname{dim} h\left(S O(n)_{\text {diag }}\right)\right)$ live on the subgroup graphs [24] $\cup_{i=1}^{M} K_{m_{i}}$. For example, the construction $S O(m) \times$ $S O(\operatorname{dim} S O(m))_{\tau}, m<n$ lives on the graph $K_{m} \cup\left(K_{1}\right)^{m-n}$, with

$$
t^{(0)}(i j l)=\left\{\begin{array}{cc}
-1 & \text { when } i, j, l \text { are points in } K_{m} \\
0 & \text { otherwise }
\end{array} .\right.
$$

4. The $\mathrm{KS} \mathrm{N}=1$ subgroup construction (B.4) on the KS subgroups $S O(n)_{\text {diag }}^{K S} \times S O\left(\operatorname{dim} h\left(S O(n)_{\text {diag }}\right)\right)$ also live on the subgroup graphs. For example, the construction $S O(m)^{K S} \times$ $S O(\operatorname{dim} S O(m))_{\tau}, m<n$ lives on the graph $K_{m} \cup\left(K_{1}\right)^{m-n}$, with

$$
t^{(0)}(i j l)=\left\{\begin{array}{cc}
-1 & \text { when at least two of the points } i, j, l \text { are in } K_{m} \\
0 & \text { otherwise }
\end{array} .\right.
$$

5. Other known constructions, which are tensor products of the constructions above, live on the unions of their corresponding graphs.

6. The quadratic deformation $\operatorname{Cartan}\left(S O(3)_{x} \times S O(3)_{2}\right)_{N=1}^{\#}$ in (6.13), which contains the subgroup constructions $S O(2) \times\left(\right.$ one free fermion) and $h^{K S}=S O(2)$, also lives on the subgroup graph $K_{2} \cup K_{1}$ with continuous $t^{(0)}=\tilde{t}$.

\footnotetext{
${ }^{* *}$ Curiously, the relevant known constructions in Appendix B are in the gauge (8.6), and we have chosen the deformation representative (6.13) in this gauge as well. Automorphic copies of these constructions are easily generated with (6.9).
} 
Since the generic construction is irrational, we may be certain that other constructions on these graphs, and the constructions that live on other graphs, are generally new.

This classification parallels that of $S O(n)_{\text {diag }}$, with the following differences:

a. Except for the complete graphs and the fundamental coset graphs, the constructions on affine-Sugawara nested graphs [24] are not identifiable as known superconformal constructions because the SME is not generally K-conjugation covariant. Similarly, the constructions on the affine-Virasoro nested graphs [24] are not related by Kconjugation, except at high level.

b. The reduced 3 -form $t(i j l)$, which lives on graph triplets of points $(i j l)$, can take multiple values on the same graph, as seen for the subgroup graphs above.

For further information on the reduced 3-form, we turn to its leading gauge-fixed equation

$$
t^{(0)}(i j l)\left[1-\left(\theta_{i j}+\theta_{i l}+\theta_{j l}\right)\right]=2 \theta_{i j} \theta_{i l} \theta_{j l} .
$$

The solutions of this equation

$$
t^{(0)}(i j l)=\left\{\begin{array}{cc}
-1 & E_{i j l}=3 \\
0 & E_{i j l}=2 \\
\text { arbitrary } & E_{i j l}=1 \\
0 & E_{i j l}=0
\end{array}\right.
$$

depend on the number of edges $E_{i j l}$ in each graph triplet of points $(i j l)$, and the list shows the third case with $E_{i j l}=1$ as the source of multiple 3-forms on a given graph. In fact, the generic graph contains at least one triplet with $E_{i j l}=1$ : We believe that the only graphs with all $E_{i j l} \neq 1$, and hence unique high-level 3-forms, are the complete graph ( $\mathrm{N}=1$ affine-Sugawara construction) and the fundamental coset graphs (KS N=1 cosets).

Although the solutions (8.15) suggest continuous 3-forms on the generic graph, it is known that continuous high-level solutions may or may not quantize at higher order [23]. This indicates a formidable program since each graph with continuous 3-forms must be studied separately for possible quantization. As an example, the 3-form of the graph $K_{2} \cup K_{1}$ does not quantize, since the graph is the deformation (6.13), but the five quantized 3 -forms

$$
t^{(0)}(123)=t^{(0)}(234)=0,\left\{\begin{array}{c}
t^{(0)}(124)=t^{(0)}(134)=0 \\
t^{(0)}(124)=t^{(0)}(134)= \pm 1 \\
t^{(0)}(124)=-t^{(0)}(134)= \pm 1
\end{array}, \quad c=\frac{9}{2}-\frac{6}{x}+\mathcal{O}\left(x^{-2}\right)\right.
$$

are obtained at the next highest order for the path graph $P_{4}$ in Fig. 3. It is likely that the central charge degeneracy of these five constructions will be lifted in the following order. 


\section{Conclusions}

We have studied the general superconformal construction on affine $g \times$ fermionic $S O(p, q)_{\tau}$. A priori, the system is described by the Virasoro master equation on this manifold, plus a set of superconformal constraints. In fact, however, the constraints imply the Virasoro master equation, so that the set of constraints is the superconformal master equation (SME), which is itself an unconstrained system.

The simple form of the general superconformal system allowed us to obtain the associated super C-function and super C-theorem, and to count the generically-expected number of superconformal constructions. A high-level expansion of the superconformal ansatz $\left(S O(n)_{d i a g} \times S O(\operatorname{dim} S O(n))_{\tau}\right)_{N=1}$ showed a large class of new, generically irrational superconformal constructions on the signed graphs of order $n$.

Although it contains only a subset of the solutions of the Virasoro master equation, the SME promises a comparably vast array of new constructions.

\section{Acknowledgment}

We thank Frank Harary for teaching us about signed graphs. 


\section{Appendix A. $\mathrm{N}=1$ stress tensor in the current-current basis}

The superconformal stress tensor $T(z)$ in (3.9) on $g_{x} \times S O(p, q)_{\tau}$ is given in the fermionic basis. Here we obtain the coefficients $L^{a b}(e, t)=L^{b a}(e, t)$ of the stress tensor

$$
\begin{aligned}
T(z) & =*\left[\sum_{A, B} L^{A B}(e, t) J_{A} J_{B}+2 \sum_{I<J} L^{A, I J}(e, t) J_{A} J_{I J}+\sum_{\substack{I<J \\
K<L}} L^{I J, K L}(e, t) J_{I J} J_{K L}\right] * \\
& ={ }^{*}\left[L^{A B}(e, t) J_{A} J_{B}+L^{A, I J}(e, t) J_{A} J_{I J}+\frac{1}{4} L^{I J, K L}(e, t) J_{I J} J_{K L}\right] *
\end{aligned}
$$

in the current-current basis $T(z)=L^{a b}{ }_{*}^{*} J_{a} J_{b}{ }_{*}^{*}$ of the Virasoro master equation (3.15). Our notation is $J_{a}=\left(J_{A}, J_{I J}\right)$ with $A=1, \cdots, \operatorname{dim} g$ and $I, J=1, \cdots, F$, where $J_{A}$ and $J_{I J}$ are respectively the "bosonic" currents in (3.7a) and the fermionic currents in (3.5). Our normal-ordering is symmetric [15], and we have adopted the natural antisymmetrization conventions

$$
L^{I J, K L}=-L^{J I, K L}=-L^{I J, L K}, L^{A, I J}=-L^{A, J I}
$$

in the second line of (A.1) in order to freely sum over the fermionic indices.

It is clear on inspection of the stress tensor in the two bases (3.9), (A.1) that

$$
L^{A B}(e, t)=\mathcal{L}^{A B}(e, t), \quad L^{A, I J}(e, t)=\sqrt{\frac{2}{\tau \psi^{2}}} \mathcal{M}^{I J A}(e, t)
$$

but some algebra is needed to obtain the form of the fermion bilinear and quartic terms in the current-current basis.

The normal-ordered $S O(p, q)_{\tau}$ current bilinear is

$$
\begin{gathered}
{ }_{*}^{*} J_{I J}(w) J_{K L}(w){ }_{*}^{*}=\lim _{z \rightarrow w}\left[J_{I J}(z) J_{K L}(w)-\frac{G_{I J, K L}}{(z-w)^{2}}\right. \\
\left.-i f_{I J, K L} R S\left(\frac{1}{z-w}+\frac{1}{2} \partial_{w}\right) J_{R S}(w)\right] \\
G_{I J, K L}=\frac{\tau \psi^{2}}{2}\left(\eta_{I K} \eta_{J L}-\eta_{I L} \eta_{J K}\right) \\
f_{I J, K L}{ }^{R S}=\sqrt{\frac{\tau \psi^{2}}{2}}\left(\eta_{J K} \delta_{I}^{[R} \delta_{L}^{S]}-\eta_{J L} \delta_{I}^{[R} \delta_{K}^{S]}-\eta_{I K} \delta_{J}^{[R} \delta_{L}^{S]}+\eta_{I L} \delta_{J}^{[R} \delta_{L}^{S]}\right)
\end{gathered}
$$

where $\eta_{I J}$ and $f_{I J, K L}{ }^{R S}$ are, respectively, the metric on the vector representation and the structure constants of $S O(p, q)$. The properties (6.3) of the text are obtained from (A.4c) with $\eta_{I J}=\delta_{I J}$ and $I, J \rightarrow i, j$. It follows from (A.4) that

$$
\begin{gathered}
{ }_{*}^{*} J_{I J} J_{K L}{ }_{*}^{*}=\frac{\tau \psi^{2}}{2}\left\{-{ }_{\circ}^{\circ} S_{I} S_{J} S_{K} S_{L} \stackrel{\circ}{\circ}-\frac{1}{2}\left(\eta_{I[K} K_{L] J}-(I \leftrightarrow J)\right)\right\} \\
K_{I J}(z) \equiv S_{I} \stackrel{\leftrightarrow}{\partial} S_{J}-\frac{\epsilon \eta_{I J}}{4 z^{2}} .
\end{gathered}
$$


and the relations

$$
\begin{aligned}
& \mathcal{F}^{I J}(e, t)=-\frac{\tau \psi^{2}}{4} L^{I K, J L}(e, t) \eta_{K L} \\
& \mathcal{R}^{I J K L}(e, t)=-\frac{\tau \psi^{2}}{24} L^{I[J, K L]}(e, t) \\
L^{I J, K L}(e, t)= & -\frac{4}{\tau \psi^{2}}\left[2 \mathcal{R}^{I J K L}+\frac{1}{F-2}\left[\left(\eta^{I[K} \mathcal{F}^{L] J}-(I \leftrightarrow J)\right)\right.\right. \\
& \left.\left.-\frac{1}{F-1} \eta^{I[K} \eta^{L] J} \mathcal{F}^{M N} \eta_{M N}\right]\right](e, t)
\end{aligned}
$$

are obtained by comparing the two bases (3.9) and (A.1) with (A.5). The results (3.15), (4.7), (4.8) and (4.10) of the text may be verified from the functional form $L^{a b}(e, t)$ in (A.3) and (A.6). 


\section{Appendix B. Standard $\mathrm{N}=1$ constructions}

For reference, we give a partial list of known solutions of the SME (3.16). In these solutions, the fermions are in the adjoint of simple compact $g$ so that $F=\operatorname{dim} g, I=A=$ $1, \cdots, \operatorname{dim} g$ and $\eta_{I J}=\eta_{A B}$, which is the Killing metric on $g$.

1) $\mathrm{N}=1$ affine-Sugawara construction on $g_{x} \times S O(\operatorname{dim} g)_{\tau}$

$$
\begin{gathered}
e^{A B}=\frac{\eta^{A B}}{\sqrt{k+Q_{g} / 2}}, \quad t^{A B C}=\frac{-f^{A B C}}{\sqrt{k+Q_{g} / 2}} \\
c=\frac{x \operatorname{dim} g}{x+\tilde{h}_{g}}+\frac{\operatorname{dim} g}{2}
\end{gathered}
$$

where $f^{A B C}$ are the structure constants of $g$, and the corresponding $\mathrm{N}=1$ affine-Sugawara constructions on $(h \times S O(\operatorname{dim} h)) \subset g_{x} \times S O(\operatorname{dim} g)$.

2) Non-linear SUSY [30]

$$
e^{A B}=0, \quad t^{A B C}=\frac{1}{\sqrt{Q_{g} / 2}} f^{A B C}, \quad c=\frac{\operatorname{dim} g}{2}
$$

3) $\mathrm{N}=1 \operatorname{cosets}\left(g_{x} \times g_{\tilde{h}}\right) / g_{x+\tilde{h}}[31]$

$$
\begin{gathered}
e^{A B}=\frac{Q_{g} / 2}{\sqrt{Q_{g}\left(k+Q_{g}\right)\left(k+Q_{g} / 2\right) / 2}} \eta^{A B}, \quad t^{A B C}=\frac{k}{\sqrt{Q_{g}\left(k+Q_{g}\right)\left(k+Q_{g} / 2\right) / 2}} f^{A B C} \\
c=\frac{x\left(x+3 \tilde{h}_{g}\right) \operatorname{dim} g}{2\left(x+\tilde{h}_{g}\right)\left(x+2 \tilde{h}_{g}\right)} .
\end{gathered}
$$

4) $\mathrm{KS} \mathrm{N}=1$ subgroup construction on $\left(h^{K S} \times S O(\operatorname{dim} h)\right) \subset g_{x} \times S O(\operatorname{dim} g)$ where $h^{K S} \equiv \times_{s=1}^{q}\left(h_{s}\right)_{r_{h_{s}}\left(x+\tilde{h}_{g}\right)-\tilde{h}_{h_{s}}} \times(U(1))^{p}[32]$

$$
\begin{gathered}
e^{A B}=\left\{\begin{array}{cc}
\frac{\eta^{a b}}{\sqrt{k+Q_{g} / 2}} & A=a, B=b \\
0 & \text { otherwise }
\end{array}\right. \\
t^{A B C}=\left\{\begin{array}{cc}
\frac{-f^{A B C}}{\sqrt{k+Q_{g} / 2}} & A=a, B=b, C=c \text { or } A=a, B=i, C=j \\
0 & \text { otherwise }
\end{array}\right. \\
c=\frac{3}{2} p+\sum_{s=1}^{q}\left[\frac{x+\tilde{h}_{g}-\tilde{h}_{h_{s}} / r_{h_{s}}}{x+\tilde{h}_{g}}+\frac{1}{2}\right] \operatorname{dim} h_{s}
\end{gathered}
$$

where $A, B \in g, a, b \in h, i, j \in g / h$, and $r_{h_{s}}=\psi_{g}^{2} / \psi_{h_{s}}^{2}$ is the embedding index of $h_{s} \subset g$.

5) $\mathrm{KS} \mathrm{N}=1$ coset construction $\left(g_{x} \times S O(\operatorname{dim} g)\right) /\left(h^{K S} \times S O(\operatorname{dim} h)\right)[32]$

$$
\begin{gathered}
e^{A B}=\left\{\begin{array}{cc}
\frac{\eta^{i j}}{\sqrt{k+Q_{g} / 2}} & A=i, B=j \\
0 & \text { otherwise }
\end{array}\right. \\
t^{A B C}=\left\{\begin{array}{cc}
\frac{-f^{i j k}}{\sqrt{k+Q_{g} / 2}} & A=i, B=j, C=k \\
0 & \text { otherwise }
\end{array}\right.
\end{gathered}
$$




$$
c=\frac{3 x \operatorname{dim}(g / h)}{2\left(x+\tilde{h}_{g}\right)}+\frac{f^{i j k} f_{i j k} /\left(2 \psi_{g}^{2}\right)}{x+\tilde{h}_{g}}
$$

which is the super K-conjugate partner of the KS subgroup construction (B.4).

For special $g / h$, there are also $\mathrm{N}=1$ solutions with antisymmetric $e^{A B}$ which appear in the $\mathrm{N}=2 \mathrm{KS}$ constructions [32]. 


\section{Appendix C. Linear terms in the supercurrent}

We generalize the discussion of the text to the supercurrent

$$
G(d)=G+2 d^{I} \partial S_{I}
$$

where $G$ is defined in (3.8). This is the most general operator with naive dimension $3 / 2$ which can be constructed from the currents and the fermions. The GG OPE $(3.2 \mathrm{~b})$ defines the stress tensor and central charge

$$
\begin{gathered}
T(d)=T+\mathcal{D}^{A} \partial J_{A}+i \mathcal{D}^{I J} \partial\left(\begin{array}{c}
\circ \\
\circ
\end{array} S_{I} S_{J} \text { ○ }\right) \\
\mathcal{D}^{A}=e^{A I} d^{J} \eta_{I J}, \quad \mathcal{D}^{I J}=\frac{1}{2} t^{I J K} d^{L} \eta_{K L} \\
c(d)=c-12 d^{I} d^{J} \eta_{I J}
\end{gathered}
$$

with $\mathrm{T}$ and $c$ given in (3.9) and (3.11). The generalized SME

$$
\begin{aligned}
& E^{A I}(e, t)+i\left(e^{B I} e^{C J} f_{B C}{ }^{A}+t^{I J K} e^{A L} \eta_{K L}\right) d^{M} \eta_{J M}=0 \\
& T^{I J K}(e, t)-i t^{N L[I} t^{J K] P} d^{M} \eta_{N P} \eta_{L M}=0 \\
& d^{I}=-4 \mathcal{F}^{I J} d^{K} \eta_{J K}=\left(e^{A I} e^{B J} G_{A B}+\frac{1}{2} t^{I K L} t^{J M N} \eta_{K M} \eta_{L N}\right) d^{P} \eta_{J P}
\end{aligned}
$$

follows from the TG OPE, where $E$ and $T$ are given in (3.16). This system contains all known $c$-changing $S L(2, R)$-preserving linear superconformal deformations [13].

The generalized SME (C.3) collects the superconformal solutions of the generalized Virasoro master equation [15]

$$
\begin{gathered}
\beta^{a b}(L)+i f_{c e}{ }^{(a} L^{b) c} D^{e}=0, \\
D^{a}\left(2 G_{a b} L^{b e}+f_{a b}{ }^{d} L^{b c} f_{c d}{ }^{e}\right)=D^{e} \\
c(D)=2 G_{a b}\left(L^{a b}-6 D^{a} D^{b}\right)
\end{gathered}
$$

on $g_{x} \times S O(p, q)_{\tau}$, which describes the generalized Virasoro construction $T(D)=L^{a b}{ }_{*}^{*} J_{a} J_{b}{ }_{*}^{*}+$ $D^{a} \partial J_{a}$ on this manifold. The translation to the current-current basis is

$$
D^{A}=\mathcal{D}^{A}, \quad D^{I J}=\sqrt{\frac{8}{\tau \psi^{2}}} \mathcal{D}^{I J}, \quad I<J
$$

Since it contains an equal number of equations and unknowns, the generalized SME is a "consistent ansatz" of (C.4). The special case of Mohammedi [27] can be obtained from the generalized SME by requiring the additional constraints $\mathcal{M}=\mathcal{R}=0$, as in the text.

A related superconformal system is

$$
\begin{gathered}
G(d)^{(r)}=G^{(r)}+d^{I} S_{I}^{(r)} \\
L(d)^{(m)}=L^{(m)}+\mathcal{D}^{A} J_{A}^{(m)}+\mathcal{D}_{I J}\left(\begin{array}{c}
\circ \\
\circ
\end{array} S_{I} S_{J}^{\circ} \circ\right)^{(m)}+\frac{1}{2} d^{I} d^{J} \eta_{I J} \delta_{m, 0} \\
c(d)=c
\end{gathered}
$$


with $G, T, c$ defined in (3.8),(3.9),(3.11) and $\mathcal{D}_{A}, \mathcal{D}_{I J}$ given in (C.2b). This case is described by the generalized SME

$$
E^{A I}(e, t)=T^{I J K}(e, t)=0, \quad d^{I}=-4 \mathcal{F}^{I J} d^{K} \eta_{J K}
$$

whose solutions include all known $c$-fixed linear superconformal deformations [13]. The system (C.6,7) collects the superconformal solutions of the generalized Virasoro master equation [15]

$$
\beta^{a b}(L)=0, \quad D^{a}\left(2 G_{a b} L^{b e}+f_{a b}^{d} L^{b c} f_{c d}^{e}\right)=D^{e}, \quad c(D)=c=2 G_{a b} L^{a b}
$$

on $g_{x} \times S O(p, q)_{\tau}$, which describes the generalized Virasoro construction $L(D)^{(m)}=$ $L^{a b} T_{a b}^{(m)}+D^{a} J_{a}^{(m)}+\frac{1}{2} D^{a} D^{b} G_{a b} \delta_{m, 0}$ on this manifold. 


\section{Appendix D. The $\mathrm{N}=2$ superconformal construction}

Among the OPE's of the $\mathrm{N}=2$ superconformal algebra [33], those which involve at least one supercurrent

$$
\begin{gathered}
G_{i}(z) G_{j}(w)=\frac{2 c / 3 \delta_{i j}}{(z-w)^{3}}+\left(\frac{2}{(z-w)^{2}}+\frac{1}{z-w} \partial_{w}\right) i \epsilon_{i j} J(w)+\frac{2 \delta_{i j}}{z-w} T(w)+\text { finite } \\
T(z) G_{i}(w)=\left(\frac{3 / 2}{(z-w)^{2}}+\frac{1}{z-w} \partial_{w}\right) G_{i}(w)+\text { finite } \\
J(z) G_{i}(w)=\frac{i \epsilon_{i j}}{z-w} G_{j}(w)+\text { finite }
\end{gathered}
$$

are sufficient to guarantee the full symmetry, since the remaining OPE's

$$
\begin{gathered}
T(z) T(w)=\frac{c / 2}{(z-w)^{4}}+\left(\frac{2}{(z-w)^{2}}+\frac{1}{z-w} \partial_{w}\right) T(w)+\text { finite } \\
T(z) J(w)=\left(\frac{1}{(z-w)^{2}}+\frac{1}{z-w} \partial_{w}\right) J(w)+\text { finite } \\
J(z) J(w)=\frac{c / 3}{(z-w)^{2}}+\text { finite }
\end{gathered}
$$

follow from (D.1) by Jacobi identities, as in the text. Our notation is $i, j=1,2$ and $\epsilon_{12}=-\epsilon_{21}=1$. We give only results, beginning with the supercurrents

$$
G_{i}(z)=e_{i}^{A I} J_{A}(z) S_{I}(z)+\frac{i}{6} t_{i}^{I J K}{ }_{\circ}^{\circ} S_{I}(z) S_{J}(z) S_{K}(z) \circ, \quad i=1,2
$$

which are $\left(e_{1}, t_{1}\right)$ and $\left(e_{2}, t_{2}\right)$ copies of $(3.8)$, and the $\mathrm{U}(1)$ current

$$
\begin{gathered}
J(z)=A^{A} J_{A}(z)+i B^{I J}{ }_{\circ}^{\circ} S_{I}(z) S_{J}(z) \stackrel{\circ}{\circ} \\
A^{A}=\frac{1}{2} e_{1}^{B I} e_{2}^{C J} f_{B C}{ }^{A} \eta_{I J} \\
B^{I J}=-\frac{1}{4}\left(e_{1}^{A I} e_{2}^{B J}-e_{2}^{A I} e_{1}^{B J}\right) G_{A B}-\frac{1}{8}\left(t_{1}^{I K L} t_{2}^{J M N}-t_{2}^{I K L} t_{1}^{J M N}\right) \eta_{K M} \eta_{L N}
\end{gathered}
$$

which follows from the OPE (D.1a). The coefficients (3.11) of the stress tensor in (3.9) may be written in terms of $\left(e_{1}, t_{1}\right)$ or $\left(e_{2}, t_{2}\right)$.

The $\mathrm{N}=2$ system consists of two copies of the SME

$$
E^{A I}\left(e_{i}, t_{i}\right)=T^{I J K}\left(e_{i}, t_{i}\right)=0, \quad i=1,2
$$

and the family of $\mathrm{N}=2$ constraints

$$
\begin{gathered}
\left(e_{i}^{A I} e_{1}^{B J}-\epsilon_{i j} e_{j}^{A I} e_{2}^{B J}\right) \eta_{I J}=0 \\
\left(e_{i}^{A I} e_{1}^{B J}-\epsilon_{i j} e_{j}^{A I} e_{2}^{B J}\right) G_{A B}+\frac{1}{2}\left(t_{i}^{I K L} t_{1}^{J M N}-\epsilon_{i j} t_{j}^{I K L} t_{2}^{J M N}\right) \eta_{K M} \eta_{L N}=0 \\
\left(e_{i}^{B I} e_{1}^{C J}-\epsilon_{i j} e_{j}^{B I} e_{2}^{C J}\right) f_{B C}{ }^{A}+\left(t_{i}^{I J K} e_{1}^{A L}-\epsilon_{i j} t_{j}^{I J K} e_{2}^{A L}\right) \eta_{K L}=0
\end{gathered}
$$




$$
\begin{gathered}
\left(t_{i}^{I M[J} t_{1}^{K L] N}-\epsilon_{i j} t_{j}^{I M[J} t_{2}^{K L] N}\right) \eta_{M N}=0 \\
\epsilon_{i j} e_{j}^{A I}=\frac{1}{2} e_{1}^{D J} e_{2}^{E K} e_{i}^{C I} f_{D E}^{B} f_{B C} \eta_{J K}+\frac{1}{2} e_{i}^{A K}\left(e_{1}^{B J} e_{2}^{C I}-e_{2}^{B J} e_{1}^{C I}\right) G_{B C} \eta_{J K} \\
+\frac{1}{4} e_{i}^{A K}\left(t_{1}^{J M N} t_{2}^{I P Q}-t_{2}^{J M N} t_{1}^{I P Q}\right) \eta_{M P} \eta_{N Q} \eta_{J K} \\
\epsilon_{i j} t_{j}^{I J K}=\left(\frac{1}{2} e_{2}^{B[K} t_{i}^{I J] M} e_{1}^{A L} G_{A B} \eta_{L M}+\frac{1}{4} t_{i}^{M[I J} t_{2}^{K] Q R} t_{1}^{L N P} \eta_{L M} \eta_{N Q} \eta_{P R}\right)-(1 \leftrightarrow 2) \quad(D .6 f)
\end{gathered}
$$

on the pair of $\mathrm{N}=1$ constructions. The quadratic constraints (D.6a-d) and the cubic constraints (D.6e,f) are required by the $G_{i} G_{j}$ and $J G_{i}$ OPE's respectively. We have checked that the $\mathrm{N}=2 \mathrm{KS}$ constructions [32] are solutions of this system. 


\section{References}

[1] K. Bardakci and M.B. Halpern, Phys. Rev. D3 (1971) 2493.

[2] P. Ramond, Phys. Rev. D3 (1971) 2415.

[3] V.G. Kač, Funct. Anal. App. 1 (1967) 328; R.V. Moody, Bull. Am. Math. Soc. $\underline{73}$ (1967) 217.

[4] A. Neveu and J.H. Schwarz, Nucl. Phys. B31 (1971) 86.

[5] M.B. Halpern, Phys. Rev. D4 (1971) 2398.

[6] M.B. Halpern, Phys. Rev. D12 (1975) 1684.

[7] T. Banks, D. Horn and H. Neuberger, Nucl. Phys. B108 (1976) 119.

[8] I.B. Frenkel and V.G. Kač, Inv. Math. 62 (1980) 23; G. Segal, Comm. Math. Phys. $\underline{80}$ (1981) 301.

[9] E. Witten, Comm. Math. Phys. 92 (1984) 455; G. Segal, unpublished; V.G. Knizhnik and A.M. Zamolodchikov, Nucl. Phys. B247 (1984) 83.

[10] P. Goddard, A. Kent and D. Olive, Phys. Lett. B152 (1985) 88.

[11] D. Gross, J.A. Harvey, E. Martinec and R. Rohm, Phys. Rev. Lett. $\underline{54}$ (1985) 502.

[12] P. Goddard and D. Olive, Int. J. Mod. Phys. A1 (1986) 303.

[13] J.K. Freericks and M.B. Halpern, Ann. of Phys. 188 (1988) 258; Erratum, ibid. $\underline{190}$ (1989) 212.

[14] M.B. Halpern, Ann. of Phys. 194 (1989) 247.

[15] M.B. Halpern and E. Kiritsis, Mod. Phys. Lett. A4 (1989) 1373; Erratum ibid. A4 (1989) 1797.

[16] M.B. Halpern and J.P. Yamron, Nucl. Phys. B332 (1990) 411.

[17] A.Yu Morozov, A.M. Perelomov, A.A. Rosly, M.A. Shifman and A.V. Turbiner, Int. J. Mod. Phys. A5 (1990) 803.

[18] E. Kiritsis, Mod. Phys. Lett. A4 (1989) 437; G. V. Dunne, I. G. Halliday and P. Suranyi, Phys. Lett. B213 (1988) 139.

[19] M.B. Halpern, E. Kiritsis, N.A. Obers, M. Porrati and J.P. Yamron, Int. J. Mod. Phys. A5 (1990) 2275.

[20] E. Witten, in Memorial Volume for V. Knizhnik, ed. L. Brink et al., World Scientific, 1990.

[21] M.B. Halpern and N.A. Obers, "Unitary Irrational Central Charge on Compact g. I. (Simply-Laced $\left.g_{x}\right)_{\#}^{q \geq 2}$ ", Berkeley preprint, UCB-PTH-89/35, (1989). To appear in Int. J. Mod. Phys. A 
[22] S. Schrans and W. Troost, Nucl. Phys. B345 (1990) 584.

[23] M.B. Halpern and N.A. Obers, Nucl. Phys. B345 (1990) 607.

[24] M.B. Halpern and N.A. Obers, "Graph Theory, SO(n) Current Algebra and the Virasoro Master Equation", Berkeley preprint, UCB-PTH-90/31, (1990). To appear in Comm. Math. Phys.

[25] M.B. Halpern and J.P. Yamron, "A Generic Affine-Virasoro Action", Berkeley preprint, UCB-PTH-90/22 (1990). To appear in Nucl. Phys. B

[26] A. Giveon, M.B. Halpern, E.B. Kiritsis and N.A. Obers, "Exact C-Function and C-Theorem on Affine-Virasoro Space", Berkeley preprint, UCB-PTH-90/48, (1990).

[27] N. Mohammedi, "General Super Virasoro Construction on Affine g", Trieste preprint, PRINT-90-0645 (1990)

[28] F. Harary, Michigan Math. J. 2 (1953) 142.

[29] A.B. Zamolodchikov, JETP. Lett. $\underline{43}$ (1986) 730; A.A. Tseytlin, Phys. Lett. B194 (1987) 63; J. Cardy, Phys. Rev. Lett. $\underline{60}$ (1988) 2709; A. Capelli, D. Friedan and J. Latorre, "C-Theorem and Spectral Representation", Rutgers preprint, RU-90-43 (1990)

[30] P. Di Vecchia, V.G. Knizhnik, J.L. Petersen and P. Rossi, Nucl. Phys. B253 (1985) 701; P. Goddard and D. Olive, Nucl. Phys. B257 (1985) 226; P. Windey, Comm. Math. Phys. 105 (1986) 511; I. Antoniadis, C. Bachas, C. Kounnas and P. Windey, Phys. Lett. B171 (1986) 51.

[31] P. Goddard, A. Kent and D. Olive, Comm. Math. Phys. 103 (1986) 105.

[32] Y. Kazama and H. Suzuki, Nucl. Phys. B321 (1989) 232; Phys. Lett. B216 (1989) 112.

[33] M. Ademollo et al., Phys. Lett. B62 (1976) 105; Nucl. Phys. B111 (1976) 77. 


\section{Figure Captions}

Fig.1 The signed labelled complete graphs of $\left(S O(3)_{d i a g} \times S O(3)_{2}\right)_{N=1}$.

Fig.2 KS coset constructions $=$ complete $M$-partite graphs. The case $M=2$ is shown .

Fig.3 The path graph of order 4 . 